\title{
Relationship between CT signs and the expression of miR-146a in colon cancer
}

\author{
ZIJIAN LIU ${ }^{1}$, YUZHU LI $^{2}$ and QUANXIANG LUO ${ }^{3}$ \\ ${ }^{1}$ Department of Hematology and Oncology, Yingcheng Hospital, The Second Hospital of \\ Shandong University in Zhaoyuan City; ${ }^{2}$ Department of General Surgery, The People's Hospital of Zhaoyuan City; \\ ${ }^{3}$ Imaging Center, Yingcheng Hospital, The Second Hospital of Shandong University in Zhaoyuan City, \\ Zhaoyuan, Shandong 265400, P.R. China
}

Received March 14, 2018; Accepted August 30, 2018

DOI: $10.3892 / 01.2018 .9415$

\begin{abstract}
Our study investigated the relationship between CT signs of colon cancer and the expression of miR-146a, to evaluate their predictive value in early diagnosis and of colon cancer. A total of 216 patients with colon cancer who were admitted to the Department of Hematology and Oncology in Yingcheng Hospital, the Second Hospital of Shandong University in Zhaoyuan City from January 2013 to March 2017 were selected as the study group. At the same time, 150 patients with benign colitis were also included to serve as the control group. CT scan was performed for patients in both the study and control groups. The expression of miR-146a in serum of patients in the study and control groups was detected by RT-qPCR, and of CT signs and miR-146a expression were analyzed. The relative expression level of miR-146a in peripheral blood of study group was significantly lower than that of the control group $(t=12.180$, $\mathrm{p}=0.001)$. Sensitivity and specificity of combined detection of CT and miR-146a in the study and control groups were 91.4 and $93.6 \%$, respectively, which were higher than those of the single test $(\mathrm{p}<0.05)$. Among CT signs of study group, luminal wall, cavity and diameter changes, as well as size of tumor and surrounding tissues showed no significant correlation with the expression of miR-146a ( $p>0.05)$. The expression level of miR-146a in patients with high-risk of invasion was significantly higher than that in patients with low-risk of invasion $(\mathrm{t}=4.791, \mathrm{p}=0.001)$. The expression level of miR-146a in lymph node metastasis was significantly lower than that in non-lymph node metastasis $(t=3.104, p=0.002)$. miR-146a may be involved in the development and progression of colon cancer. Combined detection of CT and miR-146a
\end{abstract}

Correspondence to: Dr Quanxiang Luo, Imaging Center, Yingcheng Hospital, The Second Hospital of Shandong University in Zhaoyuan City, 568 Hedong Road, Zhaoyuan, Shandong 265400, P.R. China

E-mail: qq67wp@163.com

Key words: colon cancer, CT signs, miR-146a, combined detection may enhance the sensitivity and specificity of the diagnosis of colon cancer, and may provide a good predictive value for invasion and metastasis of colon cancer.

\section{Introduction}

Colon cancer is a common human gastrointestinal cancer. Incidence of colon cancer is the third highest, and the mortality rate is the fifth among all gastrointestinal cancers. Colon cancer mainly affects people older than 40 years, and the incidence in men is 2 times higher than in women (1). With the changes in people's lifestyle, diet structure and surrounding environment, the incidence of colon cancer is showing an increase, and the onset age is becoming younger (2). The onset and development of colon tumor is a slow process, and is the result of colon polyps adenoma caused by colonic mucosal lesions. Without intervention treatment, colon tumor will gradually evolve into metastatic and invasive cancer (3). Early clinical symptoms of colon cancer patients are not obvious, and most patients are diagnosed at advanced stages. Surgical resection is the basic treatment for colon cancer. The 5-year survival rate of patients with Dukes stage A colon cancer after radical operation is approximately $90 \%$, while the 5-year survival rate of patients in Dukes stage D is as low as 5\% (4,5). At present, there are many reports on the occurrence, development and treatment of colon cancer, but specificity of the early diagnosis is still low (6) Therefore, it is particularly important to investigate the imaging features and biomarkers that are related to tumor growth and evolution.

CT examination is effective for the diagnosis of colon cancer. CT scan can be used to clearly observe the morphological changes within the colon cavity to understand the thickening and prominence of intestinal wall, so as to reveal the affected surrounding tissue (7). miR-146a is abnormally expressed in a variety of malignant tumors, and its single nucleotide polymorphism Rs2910164GC is closely related to tumor susceptibility (8). At present, there is no report on the expression and diagnostic value of miR-146a in colon cancer. This study aimed to investigate the correlations between CT signs and miR-146a expression in colon cancer as well as to explore their diagnostic value for colon cancer. 
Table I. Primer sequences of miR-146a and U6.

\begin{tabular}{lcc}
\hline Genes & Forward & Reverse \\
\hline$m i R-146 a$ & 5'-GCAGGGTCCGAGGTATTCG-3' & 5'-CGCGTGAGAACTGAATTCCAT-3' \\
U6 & 5'-CTCGCTTCGGCAGCACA-3' & 5'-AACGCTTCACGAATTTGCGT-3'
\end{tabular}

\section{Patients and methods}

General information. A total of 216 patients with colon cancer who were admitted to the Department of Hematology and Oncology in Yingcheng Hospital, the Second Hospital of Shandong University in Zhaoyuan City (Zhaoyuan, China) from January 2013 to March 2017 were selected as the study group. At the same time, 150 patients with benign colitis were also included to serve as the control group. The study group included 125 males and 91 females, and the age ranged from 35 to 61 years. The control group included 94 males and 56 females, and the age ranged from 31 to 59 years. According to the $2010 \mathrm{WHO}$ histopathological classification of gastrointestinal cancer (9), patients were divided into different groups. There were 113 cases of ulcerative type, 56 cases of mass type and 47 cases of invasive type. Clinical stage: 73 cases in stage $\mathrm{T} 1,70$ cases in stage T2, 47 cases in stage T3 and 26 cases in stage T4. Pathological differentiation: 126 cases were well differentiated, 59 cases were moderately differentiated, and 31 cases were poorly differentiated. Inclusion criteria: patients confirmed by clinical endoscopy and histopathological examination; patients who could hold their breath at least $30 \mathrm{sec}$ during CT scan; patients older than 18 years; patients who signed informed consent. Exclusion criteria: patients who had a history of malignant tumors; patients who had a history of surgery, chemotherapy and radiotherapy; patients with liver and kidney dysfunction; patients with systemic dysfunction; patients with a family history of mental illness or psychosis.

This study was approved by the Ethics Committee of Yingcheng Hospital, the Second Hospital of Shandong University in Zhaoyuan City. Signed informed consents were obtained from the patients or guardians.

Main instruments and reagents. LightSpeed VCT (GE Healthcare, Chicago, IL, USA); ABI PRISM 7300 fluorescence quantitative PCR instrument (Beckman Coulter, Inc., Brea, CA, USA); fluorescence quantitative PCR kit miRCURY LNA Universal RT microRNA PCR (Shanghai Yu Bo Biotechnology Co., Ltd., Shanghai, China); TRIzol kit (Invitrogen: Thermo Fisher Scientific, Inc., Carlsbad, CA, USA), miR-146a fluorescence quantitative PCR kit (Beckman Coulter, Inc.), SmartSpec Plus spectrophotometer (Hitachi, Tokyo, Japan); all primers were synthesized by GenScript (Nanjing, China). Primer sequences are listed in Table I.

Imaging examination. CT scan was performed with LightSpeed VCT and AW4.4 workstation. Patients were fixed in supine position, and CT scan was performed under calm breath or breath holding. Abdomen was scanned from the top of diaphragm to pubic bone. Middle and lower abdomen were also scanned for some patients. Scanning parameter: tube voltage,
90-120 kV; tube current, 150-250 MA; matrix, 512x512; pitch, 1.0; scanning layer thickness, $8 \mathrm{~mm}$; reconstruction thickness, $0.75 \mathrm{~mm}$. Contrast enhancement was performed with iohexol contrast medium which was injected using a high pressure syringe at a speed of $3 \mathrm{ml} / \mathrm{sec}$. Arterial phase, portal phase and parenchymal phase were scanned by automatic triggering method and scanning was performed at 30 and $80 \mathrm{sec}$ after arterial phase. Original data were retrospectively reconstructed. Axial image data were obtained and stored in the AW4.4 workstation for review by two chief radiologists using evaluation criteria described by Benson et al (10).

$R T$-qPCR. Peripheral blood $(5 \mathrm{ml})$ was extracted from patients in both the study and control groups and stored in EDTA-K2 anti-coagulant tube. Peripheral blood mononuclear cells (PBMCs) were isolated using lymphocyte separation fluid, and were washed twice with PBS and stored for subsequent experiments. Total RNA was extracted from PBMCs using the TRIzol kit according to the manufacturer's instructions. After RNA extraction, the purity of total RNA was determined using a $1 \%$ agarose gel electrophoresis and absorbance of RNA was measured with a SmartSpec Plus Spectrophotometer. Reverse transcription was performed to synthesize cDNA using the following conditions: $16^{\circ} \mathrm{C}$ for $30 \mathrm{~min}, 42^{\circ} \mathrm{C}$ for $30 \mathrm{~min}$ and $85^{\circ} \mathrm{C}$ for $5 \mathrm{~min}$. Synthesized cDNA samples were stored at $-20^{\circ} \mathrm{C}$ before use. PCR reaction system was: $10 \mu \mathrm{l}$ of SYBR-Green mix, $1 \mu 1$ of PCR primer mix, $5 \mu 1$ of diluted cDNA template and $4 \mu \mathrm{l}$ of RNase-free water. PCR reaction conditions were: $95^{\circ} \mathrm{C}$ for $10 \mathrm{~min}$, followed by 35 cycles of $95^{\circ} \mathrm{C}$ for $15 \mathrm{sec}, 60^{\circ} \mathrm{C}$ for $1 \mathrm{~min}$. Data analysis was performed using the software provided by the manufacturer with U6 as endogenous control. Relative expression level of miR-146a was expressed as $2^{-\Delta \Delta \mathrm{Cq}}(11)$. In the diagnosis of colon cancer using serum miR-146a, the median serum level of miR-146a was set as cut-off score, values lower than the median were set as positive and values higher than the median were set as negative.

Statistical analysis. SPSS 19.0 (Tianjin Ksoft Tech, Co., Ltd., Tianjin, China) was used for all statistical analyses. Measurement data are expressed as means \pm standard deviation (means $\pm \mathrm{SD}$ ), and data that met normal distribution were compared using t-test. Count data were expressed as percentage and compared by Chi-square test. Comparisons among multiple groups were performed by one-way analysis of variance followed by post hoc test (Least Significant Difference). $\mathrm{P}<0.05$ indicated a difference with statistical significance.

\section{Results}

There were no significant differences in age, sex, alanine aminotransferase (ALT), aspartate aminotransferase (AST), 
Table II. Comparison of baseline data between the study and control groups.

\begin{tabular}{lcccc}
\hline Items & $\begin{array}{c}\text { Study } \\
(\mathrm{n}=216)\end{array}$ & $\begin{array}{c}\text { Control } \\
(\mathrm{n}=150)\end{array}$ & $\mathrm{t} / \chi^{2}$ & P-value \\
\hline Age (years) & $50.23 \pm 7.53$ & $48.81 \pm 8.16$ & 1.714 & 0.087 \\
Gender & & & 0.847 & 0.387 \\
$\quad$ Male & 125 & 94 & & \\
Female & 91 & 56 & & \\
ALT (U/l) & $64.28 \pm 31.28$ & $59.65 \pm 33.61$ & 1.351 & 0.177 \\
AST (U/l) & $71.37 \pm 35.49$ & $68.22 \pm 40.28$ & 0.789 & 0.430 \\
r-GT (U/l) & $49.16 \pm 25.46$ & $45.82 \pm 27.19$ & 1.200 & 0.230 \\
TBil (mol/l) & $16.27 \pm 8.16$ & $15.07 \pm 8.02$ & 1.393 & 0.164 \\
Glu (mmol/l) & $6.12 \pm 1.29$ & $5.87 \pm 1.15$ & 1.905 & 0.057 \\
\hline
\end{tabular}

ALT, alanine aminotransferase; AST, aspartate aminotransferase; r-GT, r-glutamyl transferase; TBil, total bilirubin; Glu, glucose.

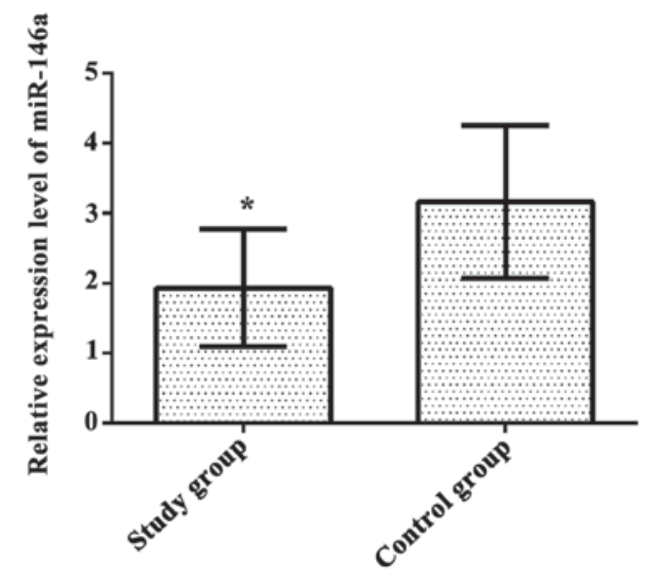

Figure 1. Expression level of miR-146a between the study and control groups. ${ }^{*} \mathrm{P}<0.001$, compared with the control group.

r-glutamyl transferase (r-GT), total bilirubin (TBil) and blood glucose (Glu) between study group and control group $(\mathrm{p}<0.05$, Table II).

Comparison of the expression level of miR-146a between the study and control groups. Relative expression level of miR-146a in study group was $1.93 \pm 0.84$ and that in the control group was 3.16 \pm 1.09 . Relative expression level of miR-146a in the study group was significantly lower than that in the control group $(\mathrm{t}=12.180, \mathrm{p}=0.001$, Fig. 1$)$.

Diagnostic efficacy of CT and miR-146a in the two groups. Diagnostic sensitivity and specificity of CT examination alone in the study and control groups were 75.6 and $87.3 \%$, respectively. Sensitivity and specificity of miR-146a alone were 72.4 and $76.5 \%$, respectively. Sensitivity and specificity of combined detection of CT and miR-146a in the study and control groups were 91.4 and $93.6 \%$, respectively. The combined diagnosis significantly improved the diagnostic sensitivity and specificity $(\mathrm{p}<0.04$, Table III).
Table III. Diagnostic efficacy of CT and miR-146a in the two groups (\%).

\begin{tabular}{lcc}
\hline Items & Sensitivity & Specificity \\
\hline CT & 75.6 & 87.3 \\
miR-146a & 72.4 & 76.5 \\
Combined diagnosis $^{\mathrm{a}}$ & $91.4^{\mathrm{a}}$ & $93.6^{\mathrm{a}}$ \\
$\chi^{2}$ & 12.388 & 13.149 \\
P-value & 0.001 & 0.001 \\
\hline
\end{tabular}

${ }^{\mathrm{a}} \mathrm{P}<0.05$, compared with $\mathrm{CT}$ or miR-146a alone.

Table IV. Correlation between CT signs and relative expression level of miR-146a in the study group (means $\pm \mathrm{SD}$ ).

\begin{tabular}{|c|c|c|c|c|}
\hline CT signs & $\mathrm{n}$ & $\begin{array}{l}\text { Relative expression } \\
\text { level of miR-146a }\end{array}$ & $\mathrm{t}$ & P-value \\
\hline Luminal wall changes & & & 1.570 & 0.117 \\
\hline Thickening & 168 & $2.16 \pm 0.84$ & & \\
\hline Non-thickening & 48 & $1.95 \pm 0.73$ & & \\
\hline Cavity changes & & & 0.931 & 0.352 \\
\hline Stenosis & 79 & $1.99 \pm 0.81$ & & \\
\hline Non-stenosis & 137 & $1.89 \pm 0.73$ & & \\
\hline Diameter changes & & & 1.400 & 0.163 \\
\hline $\begin{array}{l}\text { Non-thickening } \\
\text { or thinning }\end{array}$ & 30 & $2.37 \pm 1.19$ & & \\
\hline Thickening or block & 186 & $2.08 \pm 1.03$ & & \\
\hline Surrounding tissues & & & 1.379 & 0.169 \\
\hline Clear & 134 & $1.89 \pm 0.85$ & & \\
\hline Not clear & 82 & $2.05 \pm 0.79$ & & \\
\hline Invasion risk & & & 4.791 & 0.001 \\
\hline Low risk & 149 & $2.13 \pm 0.97$ & & \\
\hline High risk & 67 & $1.53 \pm 0.49$ & & \\
\hline Tumor size (cm) & & & 1.883 & 0.061 \\
\hline$\leq 5.0$ & 125 & $2.01 \pm 1.16$ & & \\
\hline$>5.0$ & 91 & $1.75 \pm 0.73$ & & \\
\hline $\begin{array}{l}\text { Lymph node } \\
\text { metastasis }\end{array}$ & & & 3.104 & 0.002 \\
\hline No & 135 & $2.25 \pm 1.37$ & & \\
\hline Yes & 81 & $1.73 \pm 0.81$ & & \\
\hline
\end{tabular}

Correlation between CT signs and relative expression level of miR-146a in the study group. Among all CT signs of the study group, luminal wall, cavity and diameter changes, as well as size of tumor and surrounding tissues showed no significant correlation with the expression of miR-146a ( $p>0.05)$. The expression level of miR-146a in high-risk invasion was significantly higher than that in low-risk invasion $(\mathrm{t}=4.791, \mathrm{p}=0.001)$. The expression level of miR-146a in lymph node metastasis was significantly lower than that in non-lymph node metastasis $(\mathrm{t}=3.104, \mathrm{p}=0.002$, Table IV). 


\section{Discussion}

Colon cancer is one of the most common types of malignant tumors in the digestive tract that affects 1.1 million new cases and causes approximately 500,000 deaths every year worldwide (12). Colon cancer at early stages usually shows no obvious symptoms, most patients are diagnosed at advanced stages and the best timing for treatment is missed. Therefore, early diagnosis and treatment is critical for the survival of colon cancer patients (13). The development of colon cancer is a complex and multi-stage process with multiple genes involved. At present, the pathogenesis of colon cancer is still unclear (14). Kulikov et al (15) have shown that the occurrence of colon cancer experiences normal mucosa, adenoma and cancer in 3 periods within 10-15 years. Early diagnosis and treatment can significantly improve the survival of patients (16). Therefore, prediction of the presence of tumor and its degree has great significance for the early diagnosis and treatment of colon cancer.

With the advantages of non-invasive nature, simple operation, clear images and high resolutions, CT scan is effective in the diagnosis of colon cancer. CT scan can be completed in one breath; Thus, this technique has been widely used in clinical practices (17). Diagnostic value of CT is mainly reflected by its morphological information. CT images can comprehensively and clearly show the gastrointestinal lesions. CT scan can be used to clearly reveal the luminal wall, cavity and diameter changes, as well as size of the tumor, conditions of surrounding tissues and lymph node metastasis to facilitate the development of preoperative programs and localization of tumors $(18,19)$. miRNAs play an important regulatory role in various cellular processes such as cell proliferation, differentiation and apoptosis. miRNAs regulate the expression of oncogenes or tumor suppressor genes to play a role in occurrence, development, invasion and metastasis of many types of tumors, and can serve as targets for the treatment of cancers (20). miR-146a plays a different role in a variety of malignant tumors, and is involved in tumorigenesis, tumor development, invasion and metastasis (21). Results of this study showed that the sensitivity of combined detection of CT and miR-146a was $91.4 \%$ and specificity was $93.6 \%$. Combined diagnosis significantly improved the diagnostic sensitivity and specificity of colon cancer. The relative expression level of miR-146a in the study group was significantly lower than that of the control group, suggesting that miR-146a may be involved in the occurrence and development of colon cancer. The expression level of miR-146a in patients with high-risk of invasion was significantly higher than that in patients with low-risk of invasion, and the relative expression level of miR-146a in patients with lymph node metastasis was significantly lower than that in patients without lymph node metastasis. Diagnosis using CT signs and relative expression level of miR-146a showed high consistency, indicating that the combined diagnosis using CT signs and miR-146a has good predictive values for the metastasis of colon cancer. Consistently, Shomali et al (22) showed that miR-146a is downregulated in gastric cancer to cause the downregulation of the expression of UHRF1, thereby activating the demethylation of CDH4, SLIT3 and RUNX3 promoters and further participating in the development of gastric cancer. miR-146a is associated with lymph node metastasis in gastric cancer, and serum miR-146a may be used as a diagnostic marker for gastric cancer invasion and lymph node metastasis.

Pathological examination is the main method used for the diagnosis of cancer, and the accuracy is up to $99 \%$. However, there are also some shortcomings. Pathological examination is invasive, and lymph node histological examination may miss minor metastasis. Pathological examination is also affected by subjective factors of pathologists, so accurate staging and typing of tumor tissue cannot be achieved (23). In this study, CT signs and miR-146a expression were combined to improve the early diagnosis of colon cancer. However, more clinical confirmations are still needed.

In summary, miR-146a may be involved in the occurrence and development of colon cancer. Combined detection of CT and miR-146a may improve the sensitivity and specificity of the diagnosis of colon cancer and the prediction of the invasion and metastasis of colon cancer.

\section{Acknowledgements}

Not applicable.

\section{Funding}

No funding was received.

\section{Availability of data and materials}

The datasets used and/or analyzed during the present study are available from the corresponding author on reasonable request.

\section{Authors' contributions}

ZL drafted the manuscript and performed the imaging examination. YL revised the manuscript and was also involved in the conception and design of the study. QL was responsible for RT-qPCR. All authors read and approved the final manuscript.

\section{Ethics approval and consent to participate}

The study was approved by the Ethics Committee of Yingcheng Hospital, the Second Hospital of Shandong University in Zhaoyuan City (Zhaoyuan, China). Signed informed consents were obtained from the patients or guardians.

\section{Patient consent for publication}

Not applicable.

\section{Competing interests}

The authors declare that they have no competing interests.

\section{References}

1. Viehl CT, Weixler B, Guller U, Dell-Kuster S, Rosenthal R, Ramser M, Banz V, Langer I, Terracciano L, Sauter G, et al: Presence of bone marrow micro-metastases in stage I-III colon cancer patients is associated with worse disease-free and overall survival. Cancer Med 6: 918-927, 2017. 
2. Manjelievskaia J, Brown D, McGlynn KA, Anderson W, Shriver CD and Zhu K: Chemotherapy use and survival among young and middle-aged patients with colon cancer. JAMA Surg 152: 452-459, 2017.

3. Kontovounisios C, Tan E, Pawa N, Brown G, Tait D, Cunningham D, Rasheed S and Tekkis P: The selection process can improve the outcome in locally advanced and recurrent colorectal cancer: Activity and results of a dedicated multidisciplinary colorectal cancer centre. Colorectal Dis 19: 331-338, 2017.

4. Asklid D, Segelman J, Gedda C, Hjern F, Pekkari K and Gustafsson UO: The impact of perioperative fluid therapy on short-term outcomes and 5-year survival among patients undergoing colorectal cancer surgery - a prospective cohort study within an ERAS protocol. Eur J Surg Oncol 43: 1433-1439, 2017.

5. Petrelli F, Tomasello G, Borgonovo K, Ghidini M, Turati L, Dallera P, Passalacqua R, Sgroi G and Barni S: Prognostic survival associated with left-sided vs right-sided colon cancer: A systematic review and meta-analysis. JAMA Oncol 3: 211-219, 2016.

6. Liang Q, Chiu J, Chen Y, Huang Y, Higashimori A, Fang J, Brim H, Ashktorab H, Ng SC, Ng SSM, et al: Fecal bacteria act as novel biomarkers for noninvasive diagnosis of colorectal cancer. Clin Cancer Res 23: 2061-2070, 2017

7. Lahaye M, Lambregts D, Nerad E, Bakers F, Beets G and Beets-Tan R: Clinical impact of MRI vs computed tomography in the diagnostic work-up of colon cancer patients. Eur J Cancer 72 S54, 2017.

8. Hao X, Xia L, Qu R, Yang X, Jiang M and Zhou B: Association between miR-146a rs2910164 polymorphism and specific cancer susceptibility: An updated meta-analysis. Fam Cancer: Nov 10, 2017 (Epub ahead of print).

9. Edited by Bosman FT, Carneiro F, Hruban RH and Theise ND (eds): WHO Classification of Tumours of the Digestive System. Vol 3. 4th edition. International Agency for Research on Cancer, Lyon, 2010.

10. Benson AB III, Venook AP, Cederquist L, Chan E, Chen YJ, Cooper HS, Deming D, Engstrom PF, Enzinger PC, Fichera A, et al: Colon Cancer, Version 1.2017, NCCN Clinical Practice Guidelines in Oncology. J Natl Compr Canc Netw 15: 370-398, 2017.

11. Livak KJ and Schmittgen TD: Analysis of relative gene expression data using real-time quantitative PCR and the 2(-Delta Delta C(T)) Method. METHODS 25: 402-408, 2001

12. Aquina CT, Mohile SG, Tejani MA, Becerra AZ, Xu Z, Hensley BJ, Arsalani-Zadeh R, Boscoe FP, Schymura MJ Noyes K, et al: The impact of age on complications, survival, and cause of death following colon cancer surgery. Br J Cancer 116: 389-397, 2017
13. Mahasneh A, Al-Shaheri F and Jamal E: Molecular biomarkers for an early diagnosis, effective treatment and prognosis of colorectal cancer: Current updates. Exp Mol Pathol 102: 475-483, 2017.

14. Moridikia A, Mirzaei H, Sahebkar A and Salimian J: MicroRNAs: Potential candidates for diagnosis and treatment of colorectal cancer. J Cell Physiol 233: 901-913, 2018.

15. Kulikov AV, Luchkina EA, Gogvadze V and Zhivotovsky B: Mitophagy: Link to cancer development and therapy. Biochem Biophys Res Commun 482: 432-439, 2017.

16. Fidler MM, Gupta S, Soerjomataram I, Ferlay J, Steliarova-Foucher E and Bray F: Cancer incidence and mortality among young adults aged 20-39 years worldwide in 2012: A population-based study. Lancet Oncol 18: 1579-1589, 2017.

17. Malmstrøm ML, Gögenur I, Riis LB, Hassan H, Klausen TW, Perner T, Săftoiu A and Vilmann P: Endoscopic ultrasonography and computed tomography scanning for preoperative staging of colonic cancer. Int J Colorectal Dis 32: 813-820, 2017.

18. Ren Y, Fleischmann D, Foygel K, Molvin L, Lutz AM, Koong AC, Jeffrey RB, Tian L and Willmann JK: Antiangiogenic and radiation therapy: early effects on in vivo computed tomography perfusion parameters in human colon cancer xenografts in mice. Invest Radiol 47: 25-32, 2012.

19. Baik H, Lee SM, Seo SH, An MS, Kim KH, Bae KB, Oh MK and Hong KH: Prognostic value of positron emission tomography/computed tomography for adjuvant chemotherapy of colon cancer. ANZ J Surg: Jul 7, 2017 (Epub ahead of print).

20. Weng M, Wu D, Yang C, Peng H, Wang G, Wang T and Li X: Noncoding RNAs in the development, diagnosis, and prognosis of colorectal cancer. Transl Res 181: 108-120, 2017.

21. Zhang H, Zhang Y, Yan W, Wang W, Zhao X, Ma X, Gao X and Zhang S: Association between three functional microRNA polymorphisms (miR-499 rs3746444, miR-196a rs11614913 and miR-146a rs2910164) and breast cancer risk: A meta-analysis. Oncotarget 8: 393-407, 2017.

22. Shomali N, Mansoori B, Mohammadi A, Shirafkan N, Ghasabi M and Baradaran B: MiR-146a functions as a small silent player in gastric cancer. Biomed Pharmacother 96: 238-245, 2017.

23. Govender D: Histopathology specimens: Clinical, pathological and laboratory aspects. J Clin Pathol 57: 1120, 2004. International (CC BY-NC-ND 4.0) License. 